\title{
A Portable Database of Adverse Reactions and Drug Interactions with Radiopharmaceuticals
}

\author{
Jesús Luis Gómez Perales ${ }^{1}$ and Ana Agudo Martínez ${ }^{2}$ \\ ${ }^{I}$ Nuclear Medicine Service, Puerta del Mar University Hospital, Cádiz, Spain; and ${ }^{2}$ Nuclear Medicine Service, Virgen Macarena \\ University Hospital, Sevilla, Spain
}

\begin{abstract}
Our objective was to develop a software application that allows us to easily manage a portable database of information on radiopharmaceutical interactions with drugs or other agents and on radiopharmaceutical adverse effects. Methods: The application was developed and compiled with a commercially available data management system and programming language. All data entered into the database came from the scientific literature and were accompanied by their bibliographic references. Results: We developed the database, which we have called Datinrad. To date, it contains 275 drug interactions and 44 records of adverse reactions to radiopharmaceuticals. Conclusion: Datinrad contains all the information published to date on drug-radiopharmaceutical interactions and adverse effects of radiopharmaceuticals and allows users to introduce new data from future publications. The collection of these data and their easy availability to all nuclear medicine personnel will be useful in the recognition of a possible adverse reaction or drug interaction that may alter the radiopharmaceutical biodistribution and lead to a misdiagnosis. This open-access database application is available free of charge in both English and Spanish at www.radiopharmacy.net.
\end{abstract}

Key Words: nuclear medicine; radiopharmaceutical database; drug interaction; adverse reaction

J Nucl Med Technol 2013; 41:212-215

DOI: 10.2967/jnmt.113.120626

\footnotetext{
A

radiopharmaceutical is a pharmaceutical that, when ready for use, incorporates one or more radioactive isotopes. Around $95 \%$ of radiopharmaceuticals are used for diagnostic purposes, and the rest are used for treatment of human diseases. Radiopharmaceuticals usually have no pharmacologic effects, as they are used in trace quantities. Thus, significantly unlike conventional drugs, there is no dose-response relationship for radiopharmaceuticals. Even when a radiopharmaceutical is used for therapeutic purposes, the effect achieved is not a pharmacologic consequence but

Received Jan. 25, 2013; revision accepted Mar. 27, 2013.

For correspondence or reprints contact: Jesús Luis Gómez Perales, Radiopharmacy, Nuclear Medicine, Puerta del Mar University Hospital. S.A.S. (Andalusian Health Service), Avenida Ana de Viya no. 21, 11009; Cádiz; Spain. E-mail: jesuslgomez@hotmail.com

Published online Jul. 15, 2013.

COPYRIGHT @ 2013 by the Society of Nuclear Medicine and Molecular Imaging, Inc.
}

a consequence of radioactivity. However, some exceptions exist, because radiolabeled particles present some potential mechanical issues, such as the embolic effect created by ${ }^{99 \mathrm{~m}} \mathrm{Tc}-\mathrm{macroaggregated}$ albumin and ${ }^{90} \mathrm{Y}$ resin particles or beads. In the case of ${ }^{99 \mathrm{~m} T c-m a c r o a g g r e g a t e d ~ a l b u m i n, ~}$ a sufficient number of particles need to be administered to avoid a nonuniform spatial distribution of radioactivity in lung regions; on the other hand, an excess of particles can produce acute toxicity, especially in patients with severe pulmonary hypertension. Therefore, it is important to determine the ideal number of particles for a satisfactory lung scan $(1,2)$. As for ${ }^{90} \mathrm{Y}$ resin particles or beads, which are used for therapy of primary and metastatic cancer in the liver, their particle size is $20-60 \mu \mathrm{m}$ and about 20-40 million particles per administration are needed to deliver an equivalent amount of radioactivity into the tumor. These factors increase the probability of blood stasis in the arterial vessel that supplies the tumor during therapy (embolizing effect), as well as the probability of a backflow of spheres into small collateral arteries to the stomach, duodenum, or pancreas. This phenomenon may be eliminated by applying SPECT/CT to detect accidental deposition of microspheres after application of ${ }^{99 \mathrm{~m}} \mathrm{Tc}$-macroaggregated albumin (3).

The biodistribution or pharmacokinetics of radiopharmaceuticals may be altered by a variety of drugs, disease states, and surgical procedures $(4,5)$, which can have a significant clinical impact on safety, scan interpretation, and diagnostic imaging accuracy. In their most extreme manifestations, unanticipated imaging results may even compromise the utility or accuracy of nuclear medicine studies. A recent comprehensive review (6) summarized the altered biodistribution of radiopharmaceuticals, with special emphasis on the molecular mechanisms involved. Important factors affecting the biodistribution of radiopharmaceuticals fall into 5 major categories: problems with radiopharmaceutical preparation and formulation; problems with radiopharmaceutical administration techniques and procedures; problems caused by changes in biochemical and pathophysiology; problems caused by previous medical procedures (such as surgery, radiation therapy, and dialysis); and problems caused by drug interactions.

It is also possible that an adverse reaction may occur when a radiopharmaceutical is administrated (7). It is there- 
fore important to clarify the difference between a drug interaction and an adverse reaction.

A drug interaction is defined by the Food and Drug Administration (8) as a drug-drug interaction that can lead to a change in systemic exposure, resulting in variations in the response to the coadministered drugs. Therefore, it is important to evaluate potential drug interactions before market approval and during the postmarketing period. Drug interactions with radiopharmaceuticals may lead to poor organ visualization, which can lead to misdiagnosis or the need to repeat the procedure, resulting in unnecessary irradiation of organs. Drugs or other agents such as parenteral nutrition may interfere with the nuclear medicine tests in several ways: they can directly alter the chemical identity of the radiopharmaceutical or they can affect the physiologic state of the target organs.

An adverse drug reaction is defined as any undesirable effect of a drug beyond its anticipated therapeutic effects during clinical use. An adverse reaction to radiopharmaceuticals is defined as any symptoms or response that is unexpected or unusual and undesirable. Most radiopharmaceuticals are used for diagnostic purposes and are administered in microdoses - that is, doses too low (a few micrograms) to cause pharmacologic effects but high enough to allow the cellular response to be studied. Therefore, adverse reactions to radiopharmaceuticals are generally rare, mild, and reversible without medical treatment (9-11). However, extravasated therapeutic radiopharmaceuticals can cause tissue damage because of high radiation (12-15).

For patient safety and prevention of misdiagnosis, physicians and other members of the nuclear medicine staff have to be aware of adverse reactions to radiopharmaceuticals and of drug interactions with radiopharmaceuticals. Considerable information on documented drug and radiopharmaceutical interactions has been tabulated $(4,16)$, as have adverse reactions to radiopharmaceuticals (17).

The aim of this project was to develop a personal, portable database application that allows entry, storage, and retrieval of radiopharmaceutical interactions with drugs or other agents and adverse effects of radiopharmaceuticals. Such an application can serve as a means to collect published information-both previous and new-and as an easy-to-use reference tool for nuclear medicine specialists in their daily clinical practice.

\section{MATERIALS AND METHODS}

Development of the database application, which we have called Datinrad (http://www.radiopharmacy.net/datinrad.html), included 4 stages: specifications, design, implementation, and testing (18).

\section{Database Design}

To develop Datinrad, we selected Microsoft Access, which is a relational database management system. In a relational structure, data are stored in sets of tables that are interrelated through fields having matching keys. The programming language was Visual
Basic for Applications (Microsoft). The criteria adopted to evaluate and validate the stability of the software were compliant with the Good Automated Manufacturing Practice rules (19).

\section{Implementation}

The minimum hardware requirements for Datinrad are a Pentium 4 processor (Intel) or equivalent and $2 \mathrm{~GB}$ of random-access memory. The required operating system is Windows XP Service Pack SP 2 or later (Microsoft), and the required software is Access XP or later (Microsoft) or its runtime.

\section{Data Collection}

To build the database, a review of the literature on drug interactions with radiopharmaceuticals was performed using systematic review criteria. Computerized databases such as PubMed, MEDLINE, EMBASE, SCOPUS, and Google scholar were searched using MeSH terms and keywords such as radiopharmaceutical, drug interaction, pitfall with radiopharmaceuticals, interference, and biodistribution of radiopharmaceuticals. To locate papers about adverse reactions with radiopharmaceuticals, we included in the same databases the terms adverse reaction, side effect, and radiopharmaceutical. These searches were supplemented by information from major radiopharmacy textbooks.

\section{RESULTS}

Datinrad provides 2 modules: one for interactions with radiopharmaceuticals and another for adverse reactions to radiopharmaceuticals. Every module has 2 kinds of screens: a white screen to perform queries and a red screen to add, delete, or modify records. The information must be entered into the database using a specific form (red) other than consultation (white), to ensure the integrity of data stored in the database. Figure 1 shows the user interface screen for determining interactions with radiopharmaceuticals. The screen includes a text box in which users can enter a term for a selective search by any field (radiopharmaceutical, interfering agent, study, effect, or reference). Likewise, the consultation screen of adverse reactions to radiopharmaceuticals has a text box in which users can enter a term for a selective search by any field (radiopharmaceutical, reaction, classification, or reference). Each screen has a button to change from the datasheet view to the form view and vice versa.

Our literature review identified 281 potentially relevant studies and 6 comprehensive reviews (20-25) about drug interactions with radiopharmaceuticals, along with 23 papers about adverse reactions (5 case reports and 18 studies about prevalence in different countries based on responses to questionnaires sent to nuclear medicine institutions).

To date, Datinrad contains 275 drug interactions, distributed among 158 records, and 44 records of adverse reactions to radiopharmaceuticals.

Datinrad can be transported from one computer to another using a flash drive, a compact disk, or any portable medium, provided both computers have the required operating system and software. 


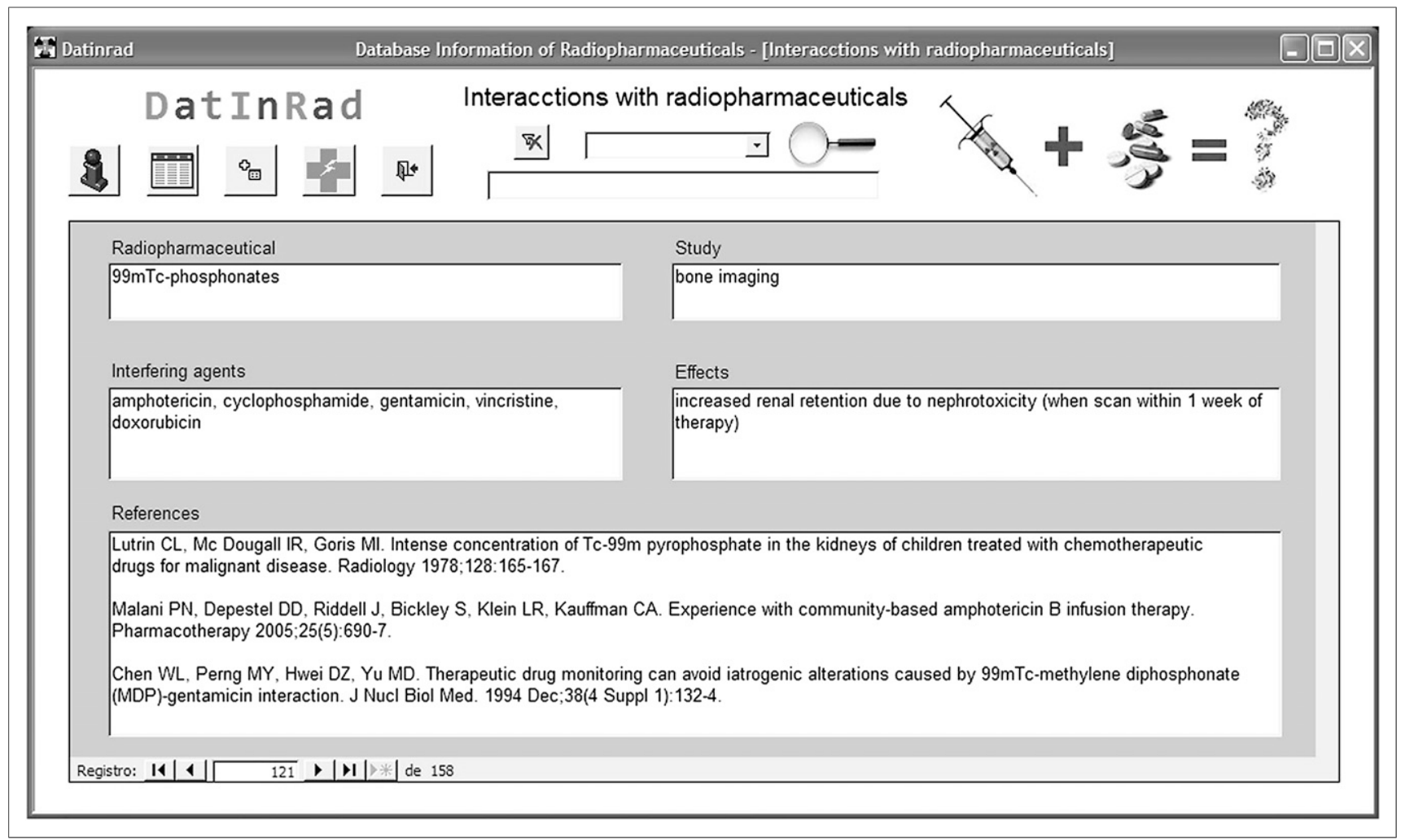

FIGURE 1. Datinrad user interface screen for interactions with radiopharmaceuticals.

\section{DISCUSSION}

Information on drug interactions with radiopharmaceuticals is becoming increasingly abundant-so much so that the nuclear medicine staff can feel overwhelmed. The challenge, therefore, is to keep all this information organized and accessible. Although the occurrence of adverse events in nuclear medicine has been rare (17,26-28), they may be seen more frequently in the future because of the administration of contrast agents in hybrid imaging and the increasing use of therapeutic radionuclides that have a significant risk of subacute and chronic toxicity (29). The consequent greater diligence required in reporting adverse events will be aided by having more easily accessible reporting systems.

There is no single system for reporting adverse events associated with radiopharmaceuticals. Different countries have different systems. For instance, the Radiopharmacy Committee of the European Association of Nuclear Medicine maintains an adverse reaction and defective product database hosted by the British Nuclear Medicine Society. However, underreporting is a recognized problem; for example, annual reports about the European Association of Nuclear Medicine database have not been published in recent years. In addition, adverse events may be described in scientific articles without being entered into a database.

In the United States, the Radiopharmaceutical Drug Information Center was developed in 1997 through a joint project between the University of Kansas Medical Center and the University of New Mexico. In connection with this international project, an article was published describing the development of the Radiopharmaceutical Information Database (RID) (30). To our knowledge, RID was the first attempt to develop a computerized database specifically for radiopharmaceutical information. However, this application is no longer available on the Internet.

All these considerations suggest that a logical framework should be created to enable review-based clinical decision making. For this reason, we aimed to develop a personal, portable database that compiles known reactions and interactions with radiopharmaceuticals from clinical and experimental evidence.

Users can choose between 2 options for updating Datinrad: in the first, users can personalize their copy of the application by adding, deleting, or modifying records; in the second, users can download an updated version that the authors will make available every year or so as they add new data appearing in the literature. These 2 options are not mutually exclusive: users who choose to personalize their copy can also download the updated database. The date of each update will be indicated on the Datinrad Web site.

\section{CONCLUSION}

Drug interactions with radiopharmaceuticals and adverse reactions to radiopharmaceutical should be better documented and reported. Collecting these data and providing all nuclear medicine staff with access to them may help decrease the incidence of adverse reactions to 
radiopharmaceuticals and prevent misdiagnoses. To this end, the database software application Datinrad might play an important role and would be particularly welcomed as a quick guide for routine daily use by nuclear medicine staff in hospitals.

Datinrad contains all the information published to date about radiopharmaceutical drug interactions and adverse reactions, besides being customizable by individual users who wish to enter new interactions or side effects that appear in the literature. Users can also download periodic updates that will be made available by the authors of the database. These compiled, easily available data may help the nuclear medicine staff to recognize a possible adverse reaction or a drug interaction that may alter the radiopharmaceutical biodistribution and lead to a misdiagnosis.

This open-access database is available in both English and Spanish, and its registration license key is free of charge. Any questions, ideas, or suggestions from nuclear medicine and radiopharmacy professionals about improving the database software will be welcomed (datinrad@ radiopharmacy.net).

\section{DISCLOSURE}

No potential conflict of interest relevant to this article was reported.

\section{REFERENCES}

1. Heck LL, Duley JW. Statistical considerations in lung imaging with Tc-99m albumin particles. Radiology. 1974;113:675-679.

2. Perales JL, García-Mendoza A. Development of a comprehensive software application for calculations in nuclear medicine and radiopharmacy. $\mathrm{J} \mathrm{Nucl} \mathrm{Med}$ Technol. 2010;38:153-162.

3. Salem R, Lewandowski RJ, Mulcahy MF, et al. Radioembolization for hepatocellular carcinoma using yttrium-90 microspheres: a comprehensive report of long-term outcomes. Gastroenterology. 2010;138:52-64.

4. Hesslewood S, Leung E. Drug interaction with radiopharmaceuticals. Eur J Nucl Med. 1994;21:348-356.

5. Sampson CB. Drugs and chemicals which affect the purity, biodistribution and pharmacokinetics of radiopharmaceuticals. J Biopharm Sci. 1990;1:381-400.

6. Vallabhajosula S, Killeen RP, Osborne JR. Altered biodistribution of radiopharmaceuticals: role of radiochemical/pharmaceutical purity, physiological, and pharmacologic factors. Semin Nucl Med. 2010;40:220-241.

7. Silindir M, Özer AY. Adverse reactions to radiopharmaceuticals (ARRP): particularly to technetium radiopharmaceuticals. FABAD J Pharm Sci. 2008;33: 109-117.

8. Drug Development and Drug Interactions. U.S. Food and Drug Administration Web site. http://www.fda.gov/Drugs/DevelopmentApprovalProcess/ DevelopmentResources/DrugInteractionsLabeling/ucm080499.htm. Accessed June 20, 2013.
9. Saha GB. Adverse reactions to radiopharmaceuticals and altered biodistribution of radiopharmaceuticals. In: Saha GB, ed. Fundamentals of Nuclear Pharmacy. New York, NY: Springer; 2004:351-353.

10. Verbruggen A, Coenen HH, Deverre JR, et al. Guideline to regulations for radiopharmaceuticals in early phase clinical trials in the EU. Eur J Nucl Med Mol Imaging. 2008;35:2144-2151.

11. Pirmohamed M, Breckenridge AM, Kitteringham NR, Park BK. Adverse drug reactions. BMJ. 1998;316:1295-1298.

12. Castronovo FP Jr, McKusick KA, Strauss HW. Dosimetric consequences of radiopharmaceutical infiltrations. Invest Radiol. 1994;29:59-64.

13. Hoop B. The infiltrated radiopharmaceutical injection: risk considerations. J Nucl Med. 1991;32:890-891.

14. Shapiro B, Pillay M, Cox PH. Dosimetric consequences of interstitial extravasation following IV administration of a radiopharmaceutical. Eur J Nucl Med. 1987;12:522-523.

15. Bonta DV, Halkar RK, Alazraki N. Extravasation of a therapeutic dose of ${ }^{131} \mathrm{I}$ metaiodobenzylguanidine: prevention, dosimetry, and mitigation. J Nucl Med. 2011;52:1418-1422.

16. Agudo A, Gómez-Perales JL, Tirado JL. Altered biodistribution of radiopharmaceuticals caused by drug interactions [in Spanish]. ALASBIMN J. May 4, 2012. http://www.alasbimnjournal.net/contenidos/alteraciones-en-labiodistribucion-de-los-radiofarmacos-causadas-por-interacciones-medicamentosas65. Accessed June 20, 2013.

17. Silberstein EB, Ryan J, Pharmacopoeia Committee of the Society of Nuclear Medicine. Prevalence of adverse reactions in nuclear medicine. J Nucl Med. 1996;37:185-92.

18. Rosen II. Writing software for the clinic. Med Phys. 1998;25:301-309.

19. GAMP 5. Tampa, FL: International Society for Pharmaceutical Engineering; 2008.

20. Lentle BC, Scott JR. Iatrogenic alterations in radionuclide biodistribution. Semin Nucl Med. 1979;9:131-143.

21. Hladik WB, Nigg NK, Rhodes BA. Drug induced changes in the biologic distribution of radiopharmaceuticals. Semin Nucl Med. 1982;12:184-218.

22. Hladik WB, Ponto JA, Lentle BC, Laven DL. Iatrogenic alterations in the biodistribution of radiotracers as a result of drug therapy: reported instances. In: Hladik WB, Saha BG, Study KT, eds. Essentials of Nuclear Medicine Science. New York, NY: Williams and Wilkins; 1987.

23. Leung E, Hesslewood S. Drug interactions with radiopharmaceuticals. Pharm J. 1992;248:47-49.

24. Sampson CB. Adverse reactions and drug interactions with radiopharmaceuticals. Drug Saf. 1993;8:280-294.

25. Santos-Oliveira R. Radiopharmaceutical drug interactions. Rev Salud Publica (Bogota). 2008;10:477-487.

26. Hesslewood SE. European system for reporting adverse reactions to and defects in radiopharmaceuticals: annual report 2000. Eur J Nucl Med Mol Imaging. 2002;29:BP13-BP19.

27. Hesslewood SR, Keeling DG, Radiopharmacy Committee of European Association of Nuclear Medicine. Frequency of adverse reactions to radiopharmaceuticals in Europe. Eur J Nucl Med. 1997;24:1179-1182.

28. Kusakabe K, Okamura T, Kasagi K, et al. Subcommittee for Safety Issues of Radiopharmaceuticals, Medical Science, and Pharmaceutical Committee, Japan Radioisotope Association. The 27th Report on Survey of the Adverse Reaction to Radiopharmaceuticals (the 30th survey in 2004) [in Japanese]. Kaku Igaku. 2006;43:23-35.

29. Hesse B, Vinberg N, Berthelsen AK, Ballinger JR. Adverse events in nuclear medicine: cause for concern? Eur J Nucl Med Mol Imaging. 2012;39:782785 .

30. Blaine P, Spicer J, Generali J, Hladik W, Johnson G. Development of a radiopharmaceutical information database. J Nucl Med Technol. 1999;27:230233. 